\title{
3D radar imaging based on frequency-scanned antenna
}

\author{
Sun Zhan-shan ${ }^{\text {a) }}$, Ren Ke, Chen Qiang, Bai Jia-jun, and Fu Yun-qi \\ College of Electronic Science and Engineering, National University of Defense \\ Technology, Deyacun, Hunan Province, Changsha 410073, China \\ a)szs199252@163.com
}

Abstract: A frequency-scanned antenna is designed to achieve range, azimuth and elevation 3D imaging. The frequency-scanned antenna is the combination of substrate integrated waveguide (SIW) slot array and planar array antenna. Angle and range information can be obtained using a single frequency-scanned antenna. So the azimuth and elevation information can be obtained respectively with two orthogonal frequency-scanned antennas in the horizontal and vertical direction. Based on the captured range, azimuth and elevation information, a 3D scenario can be constructed. The frequencyscanned antennas working in the range of 24 to $25.6 \mathrm{GHz}$ have been fabricated, and imaging experiments on different scenarios have been carried out.

Keywords: frequency-scanned antenna, substrate integrated waveguide, planar array antenna, 3D radar imaging

Classification: Microwave and millimeter-wave devices, circuits, and modules

\section{References}

[1] T. Geibig, et al:: "Compact 3D imaging radar based on FMCW driven frequency-scanning antennas," IEEE Radar Conference (2016) (DOI: 10.1109/ RADAR.2016.7485168).

[2] Y. Alvarez-Lopez, et al.: "Frequency scanning based radar system," Prog. Electromagnetics Res. 132 (2012) 275 (DOI: 10.2528/PIER12071811).

[3] S. T. Yang and H. Ling: "Application of a microstrip leaky wave antenna for range-azimuth tracking of humans," IEEE Geosci. Remote Sens. Lett. 10 (2013) 1384 (DOI: 10.1109/LGRS.2013.2243401).

[4] S. T. Yang and H. Ling: "Design of a microstrip leaky-wave antenna for two-dimensional bearing tracking," IEEE Antennas Wireless Propag. Lett. 10 (2011) 784 (DOI: 10.1109/LAWP.2011.2163377).

[5] W. Mayer, et al:: "A novel direct-imaging radar sensor with frequency scanned antenna," IEEE MTT-S International Microwave Symposium Digest 3 (2003) 1941 (DOI: 10.1109/MWSYM.2003.1210538).

[6] L. Yan, et al.: "Cui, simulation and experiment on SIW slot array antennas," IEEE Microw. Wireless Compon. Lett. 14 (2004) 446 (DOI: 10.1109/LMWC. 2004.832081).

[7] Y. L. Zhang, et al:: "Analysis of guided-wave problems in substrate integrated waveguides-numerical simulations and experimental results," IEEE MTT-S International Microwave Symposium Digest 3 (2003) 2049 (DOI: 10.1109/ MWSYM.2003.1210563). 
[8] J. Liu, et al.: "Substrate Integrated Waveguide (SIW) Leaky-Wave Antenna With Transverse Slots," IEEE Trans. Antennas Propag. 60 (2012) 20 (DOI: 10. 1109/TAP.2011.2167910).

[9] T. A. Milligan: Modern Antenna Design (McGraw-Hill, 1985).

[10] R. S. Elliott and W. O'Loughlin: "The design of slot arrays including internal mutual coupling,” IEEE Trans. Antennas Propag. 34 (1986) 1149 (DOI: 10. 1109/TAP.1986.1143947).

[11] A. Shoykhetbrod, et al:: "Design of a SIW meander antenna for $60 \mathrm{GHz}$ applications," 2012 The 7th German Microwave Conference (GeMiC) (2012) 1.

[12] F. Xu, et al.: "Finite-difference frequency-domain algorithm for modeling guided-wave properties of substrate integrated waveguide," IEEE Trans. Microw. Theory Techn. 51 (2003) 2221 (DOI: 10.1109/TMTT.2003.818935).

\section{Introduction}

Three-dimensional radar imaging of a scenario has always been a meaningful research topic. In many applications, phased array, mechanically scanned antenna or multiple channels systems are the most common methods to realize 3D imaging. However, in most cases the antennas in these systems are very complex, large and expensive due to the necessary phase shifters, power distribution networks or multiple transmit-receive channels [1]. Frequency-scanned antenna is a low cost and simple method to realize electronic scanning function since the beam steers with the changing of feed frequency [1, 2, 3, 4]. Using swept frequency signal as the feed source, the main beam can scan a certain range in one dimension $[1,5]$. With two orthogonal frequency-scanned antennas, the horizontal and vertical scanning are executed, so the azimuth and elevation information can be obtained respectively.

The common types of frequency-scanned antenna include waveguide leaky wave antenna, microstrip leaky wave antenna and SIW slot antenna. In [3] and [4], the microstrip leaky wave antenna achieves range and azimuth $2 \mathrm{D}$ imaging. As the frequency is increased from 4.25 to $8 \mathrm{GHz}$, the main beam of the antenna steers from $70^{\circ}$ to $33^{\circ}$ and the antenna gain is increased from 6.6 to $12.2 \mathrm{~dB}$. It's noted that the demand of operating bandwidth is quite large and the antenna gain in the scanning range is unsteady. Besides, due to the asymmetry of the scanning range, the antenna has to tilt an angle. In [1], a 3D radar imaging system has been build based on slow wave SIW slot antenna at V-band and the scanning range is $-30^{\circ}$ to $30^{\circ}$ with the operating bandwidth $6 \mathrm{GHz}$. This system realizes $3 \mathrm{D}$ imaging with high range and angle resolution, but the cost and complexity is quite large since the broad bandwidth requirement of the system.

In this paper, we present a 3D radar imaging system based on frequencyscanned antenna. The antenna is designed by the combination of slow wave SIW feed and planar array antenna. The planar array antenna is covered on the slow wave SIW and the coupled from slots. Therefore, the aperture of the antenna is extended significantly and the antenna gain increases accordingly. Besides, according to the design of slow wave line in the SIW, the main beam can scan from $-30^{\circ}$ 
to $30^{\circ}$ with an operating bandwidth $1.6 \mathrm{GHz}$. This bandwidth is an optimization compared with other frequency-scanned antennas.

\section{Frequency-scanned antenna}

To design a low profile and light weight antenna, the SIW structure is used. The SIW can be equivalent to a conventional rectangular waveguide in planar form [6]. In this paper, round metallized via holes are used to create the electric side walls through two parallel via arrays and hence the slow wave line is formed $[6,7,8]$.

To extend the aperture of the antenna, a planar array antenna is covered on the top layer of the SIW, which can be seen in Fig. 1. Each element of the planar array consists of two rectangular patches. Refer to the coupling design methods in [9] and [10], the planar array antenna can be fed through the corresponding slots. The longitudinal propagation constant $\beta$ determines the phase-offset among the adjacent slots $[3,11,12]$. For the SIW, $\beta$ is given by

$$
\beta=2 \pi \sqrt{1-\left(\lambda / 2 a_{\text {equ }}\right)^{2}} / \lambda
$$

where $\lambda$ is the dielectric wavelength, $a_{\text {equ }}$ is the width of the equivalent waveguide and it is mainly determined by the interval of the two parallel via holes array $(a)$ and dielectric constant $\varepsilon$ [6]. Thus, the main beam direction $\theta$ of the uniform linear array can be calculated by the following equation:

$$
\theta=\sin ^{-1}\left(\beta l / k_{0} d\right)
$$

where $k_{0}$ is the free space wavenumber, $d$ is the straight-line distance of the slots and $l$ is the length of the slow wave line between adjacent slots.

To avoid grating lobes, the length $d$ is typically close to half the wavelength at the centre frequency. Refer to the formula (2), the longer the slow wave line is chosen, the larger the scanning range is. Therefore, the slow wave line is usually stretched by bending periodically [6]. However, long slow wave line will increase transmission loss. In this paper, $d$ is $\lambda / 2$ and $l$ is about $5 d$.

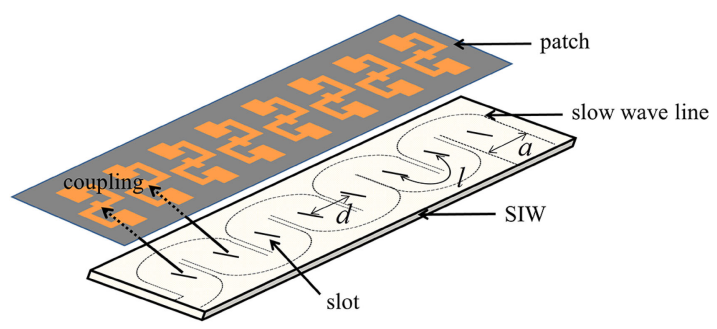

Fig. 1. Schematic diagram of frequency-scanned antenna.

With the changing of frequency, the main beam direction steers as shown in Fig. 2. Due to the limit of antenna aperture, the beam has a certain width and it determines the angle resolution, which will be illustrated in section 3 .

As shown in Fig. 3, the frequency-scanned antenna is fabricated. The size of the antenna is $148 \mathrm{~mm} \times 51 \mathrm{~mm}$ and is $5.2 \mathrm{~mm}$.

The measured results of the radiation pattern are shown in Fig. 4. As shown in Fig. 4(a), the antenna gain is about 13 to $15 \mathrm{~dB}$ as the frequency is increased from 


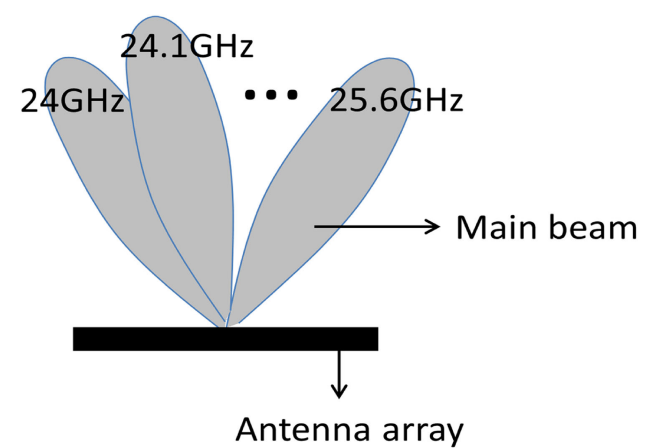

Fig. 2. Illustration of the frequency-scanned antenna pattern.

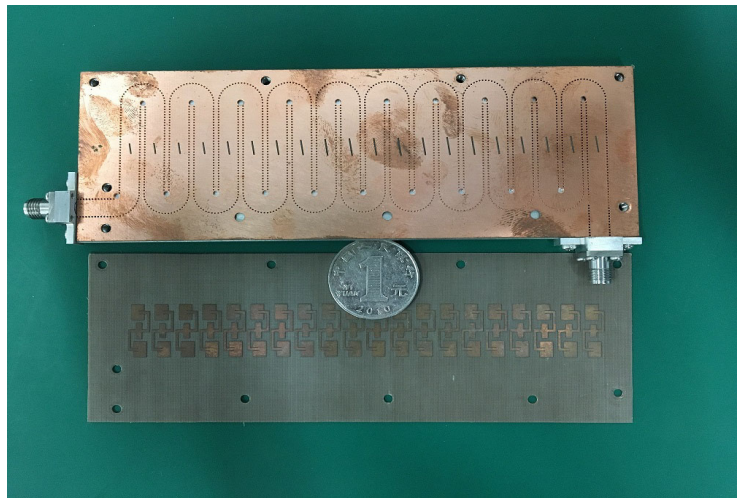

Fig. 3. Prototype of the frequency-scanned antenna.

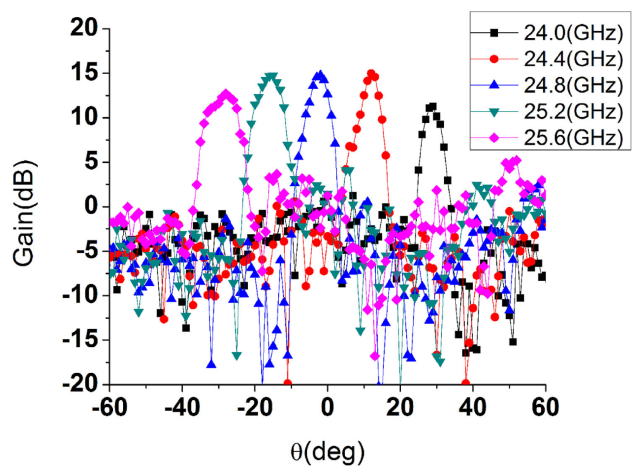

(a)

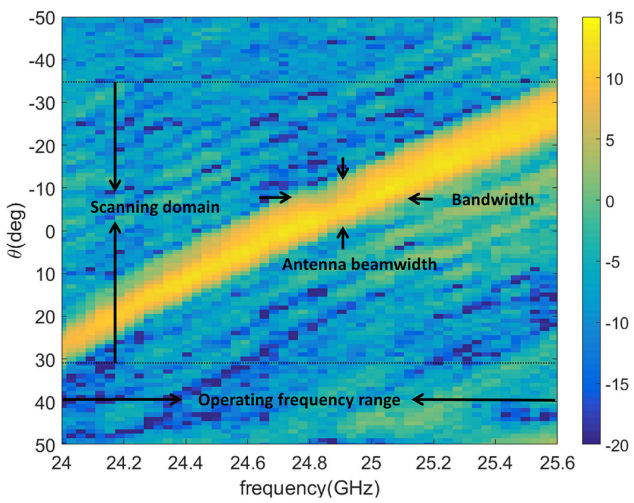

(b)

๑ IEICE 2017

DOI: $10.1587 /$ elex.14.20170503

Received May 15, 2017

Accepted May 22, 2017

Publicized June 5, 2017

Copyedited June 25, 2017 
24 to $25.6 \mathrm{GHz}$, which is taken as the operating frequency range of the radar and about $6.7 \%$ relative bandwidth. Fig. 4(b) shows the relationship of frequency and main beam direction in an intuitive way. It indicates that the scanning range is $-30^{\circ}$ to $30^{\circ}$ in the operating frequency range. The results illustrate that the antenna can obtain a large scanning range in a relative narrow bandwidth and the antenna gain is steady and relative high. In these aspects, the design of this antenna makes an great optimization compared with other frequency-scanned antennas.

\section{Imaging principle and processing algorithm}

In this paper, the transmitting antenna is the same with receiving antenna. The working principle in one dimension, horizontal scanning for example, is shown in Fig. 5. Swept frequency signal is used as the feed source of the proposed antenna. With the changing of feed frequency, the main beam steers accordingly and the radiation signal will be reflected when the main beam is pointing to targets. The frequency of received signal indicates the azimuth refer to Fig. 4(b) while the echo time indicates the range information.

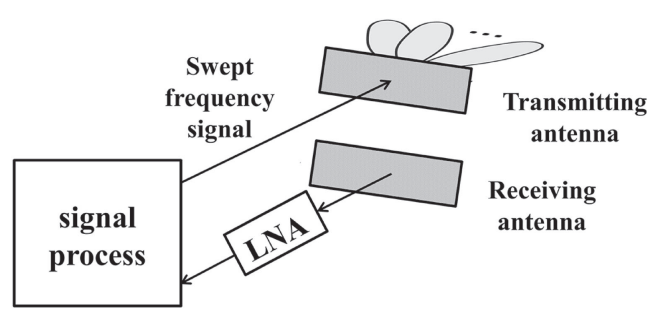

Fig. 5. The diagram of horizontal scanning.

Each target in different azimuth leads to a certain frequency response. In practice, the peaks of the frequency responses have certain bandwidth since the beamwidth of the antenna is finite [3]. The relationship between beamwidth and bandwidth can be seen in Fig. 4(b). It indicates that a particular frequency corresponds to a certain angle range due to the beamwidth and this angle range will be smaller with the decreasing of the beamwidth. Therefore, the beamwidth determines the angle resolution. To improve the angle resolution, extending the antenna aperture is an effective method.

While the frequency response is achieved, the range spectrum can be obtained by taking inverse Fourier Transform in the frequency domain. So far, the preliminary result of range and azimuth are confirmed. However, there will be a correspondence problem between range and angle since the frequency responses of multiple targets are mixed so that the range information obtained by inverse Fourier Transform is hard to match its corresponding frequency response. To solve this problem, we get the range domain by inverse Fourier Transform in the frequency domain at first, and then gate out the concerned range fragment using window functions, finally, take Fourier Transform to each fragment and obtain its corresponding frequency response respectively. Furthermore, when the transmitting and receiving antennas whose structure are the same with those in horizontal are 
placed vertically, the vertical scanning can be carried out, therefore the elevation of targets can be obtained and the resolutions of the two dimensions are identical. The working principal of vertical scanning is shown in Fig. 6.

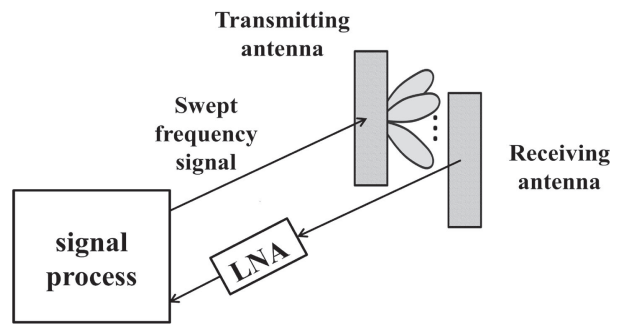

Fig. 6. The diagram of vertical scanning.

To realize $3 \mathrm{D}$ imaging of a scenario, angle and range information in two orthogonal dimensions are needed so that the azimuth and elevation with the same range are regarded as a group. However, in the case that multiple targets are located at the same range with different angle information, there will be a matching problem for the azimuth and elevation, and may lead to misjudgment. Generally, the frequency response intensity and power spectral density of different targets have certain difference, therefore the angle information in two dimensions with the same range can be matched accurately. The flow diagram of signal processing is presented in Fig. 7.

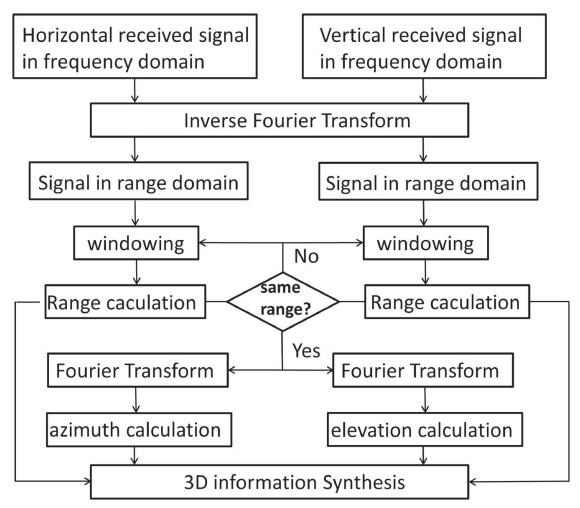

Fig. 7. Flow diagram of signal processing in this system.

\section{Experimental results}

To verify the effectiveness of radar imaging in $3 \mathrm{D}$, a measurement is carried out using a vector network analyzer (VNA) as the signal processor. Two stationary trihedrals are used as targets and the VNA collects the S21 data from $23 \mathrm{GHz}$ to $27 \mathrm{GHz}$ in 801 points to get the frequency response and the echo time of the targets. Firstly, two targets are placed at different elevation but the same range and azimuth. The experimental setup is shown in Fig. 8. 


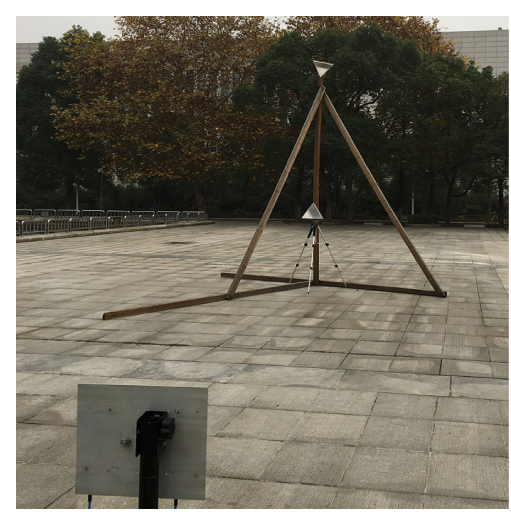

Fig. 8. Flow diagram of signal processing in this system.

In the measurement, the actual location of two targets are $\left(10 \mathrm{~m},-9.7^{\circ}, 16.7^{\circ}\right)$ and $\left(10 \mathrm{~m},-9.7^{\circ}, 1.8^{\circ}\right)$ as the form (range, azimuth, elevation). By executing the vertical scanning and horizontal scanning respectively, the measured results are shown in Fig. 9.

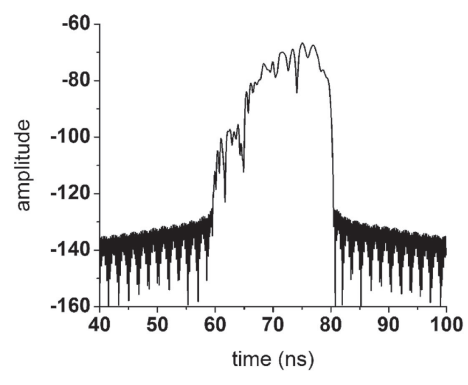

(a)

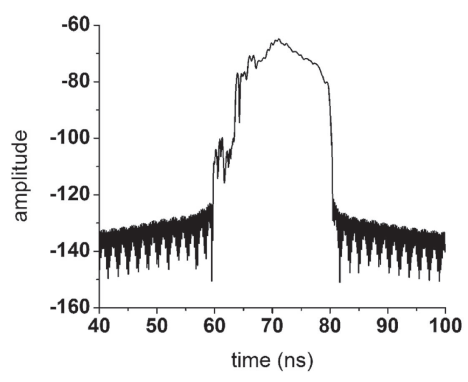

(c)

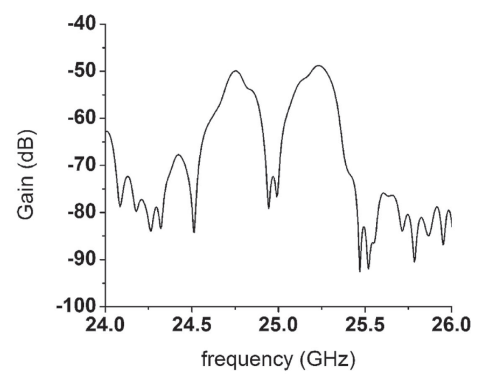

(b)

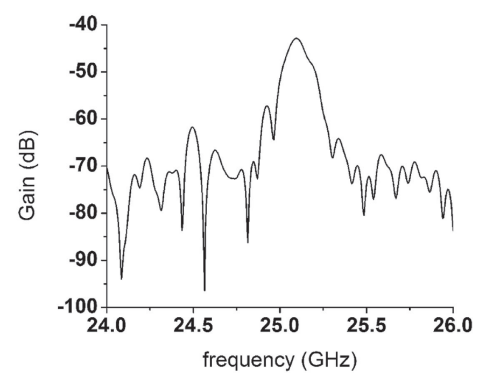

(d)

Fig. 9. (a) Time response by vertical scanning; (b) Frequency response transformed from the time response in (a); (c) Time response by horizontal scanning; (d) Frequency response transformed from the time response in (c).

Since the two targets are placed at the same range and azimuth, the time domain response and frequency domain response by horizontal scanning have only one peak, which can be seen in Fig. 9.(a), (c) and (d). The echo time indicates the range, and from Fig. 4, the angles in vertical and horizontal dimensions can be calculated. So far, the information of three dimensions is obtained and the 3D imaging result is shown in Fig. 10. 


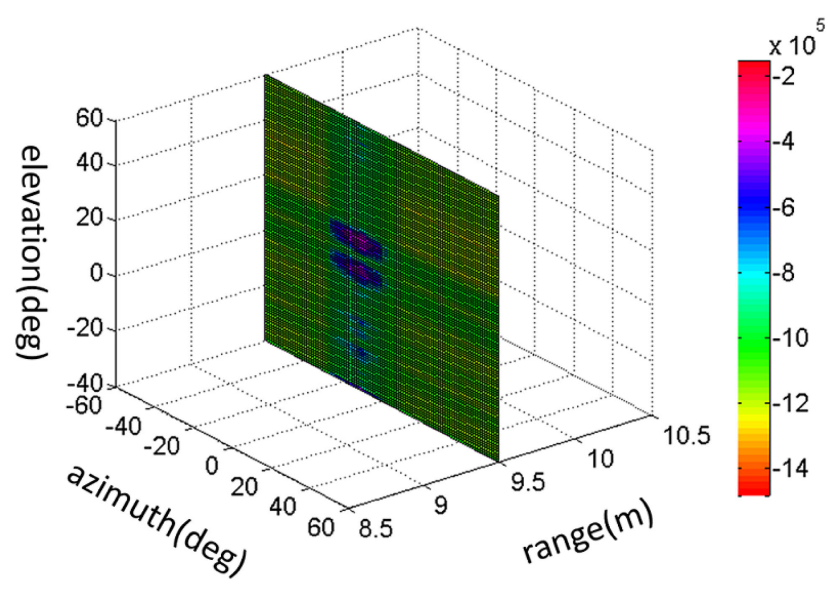

Fig. 10. 3D imaging result of situation 1 .

As shown in Fig. 10, due to the beamwidth of the antenna, the visualizations of the targets have certain profile. Since the gains and beamwidths of the antennas in different scanning angles are not exactly the same, the amplitude responses in two dimensions have some differences accordingly. As a result, the image of the target is not a round in shape, but an oval, although the resolutions in two dimensions are still identical. Generally, the geometric center of the profile is regarded as the calculated point $[3,4]$. From Fig. 10, it can be seen that the measured location of two targets are about $\left(10 \mathrm{~m},-11^{\circ}, 15^{\circ}\right)$ and $\left(10 \mathrm{~m},-11^{\circ}, 1^{\circ}\right)$. The measured results are basically the same as the actual situation.

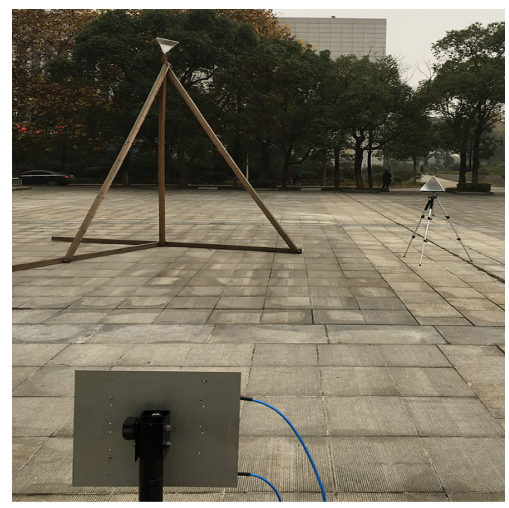

Fig. 11. Flow diagram of signal processing in this system.

And then, as shown in Fig. 11, two targets are placed at $\left(9.5 \mathrm{~m},-9.7^{\circ}, 16.7^{\circ}\right)$ and $\left(8.5 \mathrm{~m}, 13.6^{\circ}, 2^{\circ}\right)$. Similar to the previous processing method, the measured results are plotted in Fig. 12.

Similar to the signal process method of situation 1, the angles and range are calculated. Based on the flow diagram shown in Fig. 7, the angles and range information of target 1 and target 2 is synthesized into 3D respectively. The imaging result is plotted in Fig. 13 and it can be seen that the locations of two targets are $\left(9.56 \mathrm{~m},-11^{\circ}, 15^{\circ}\right)$ and $\left(8.33 \mathrm{~m}, 10^{\circ}, 1^{\circ}\right)$. 


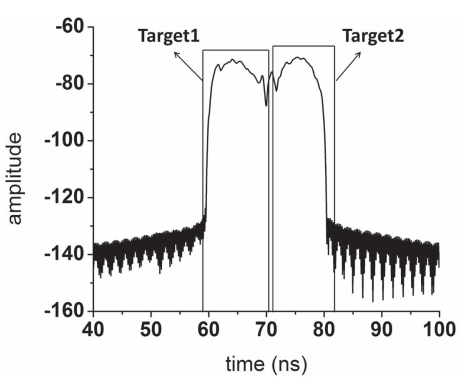

(a)

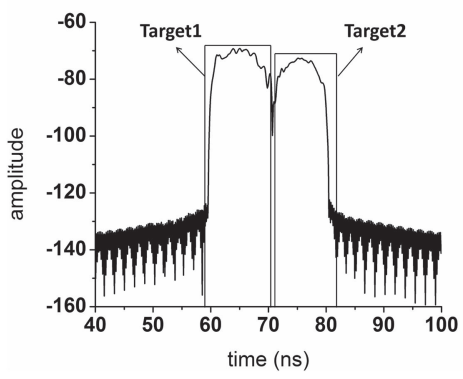

(c)

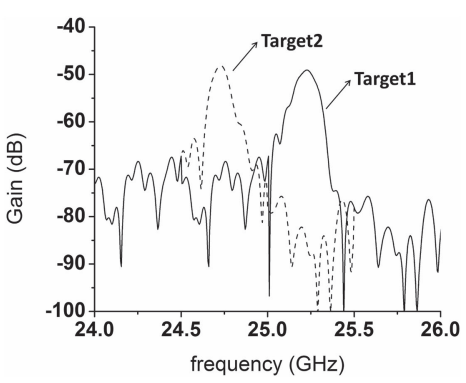

(b)

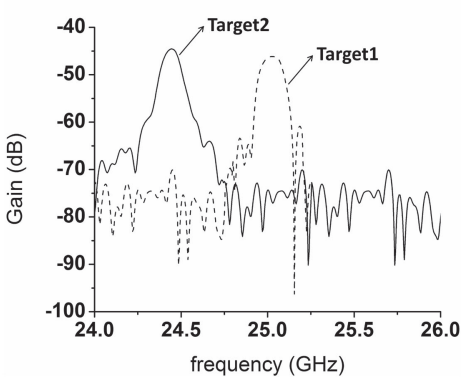

(d)

Fig. 12. Measured results of two targets at different range, azimuth and elevation. (a) Time responses by vertical scanning; (b) Frequency responses which are transformed from the time responses in (a), respectively. The dotted line represents target1 and the solid line represents target2; (c) Time responses by horizontal scanning; (d) Frequency responses which are transformed from the time responses in (c), respectively.

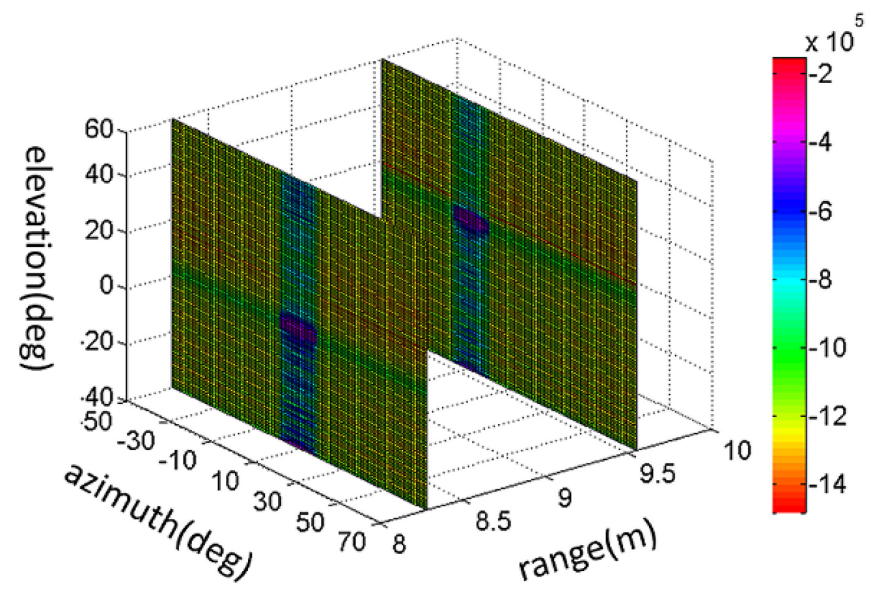

Fig. 13. 3D imaging result of situation 2 .

\section{Conclusion}

In this letter, we present a 3D imaging radar based on frequency-scanned antenna. The antenna is designed by the combination of SIW and planar array. Measured result shows that the antenna realizes a scanning range from $-30^{\circ}$ to $30^{\circ}$ in the frequency range of 24 to $25.6 \mathrm{GHz}$. Using a VNA as the signal processor, the azimuth, elevation and range information are obtained by two orthogonal frequency-scanned antennas. Two different scenarios are set and targets in different 
locations are measured. This paper illustrates that achieving 3D imaging by using the proposed frequency-scanned antenna is an efficient and simple method on account of the advantages of miniaturization, easy integration and narrow operating bandwidth. An efficient signal processing algorithm to improve the range and angle resolution is currently under development and will be applied in further research.

\section{Acknowledgments}

This work was supported by the National Natural Science Foundation of China (Grant No. 61571448). 